\title{
Vectorlike quarks and heavy colored bosons at the LHC
}

\author{
A. Deandrea* \\ Université de Lyon, F-69100 Villeurbanne, France and Université Lyon 1, CNRS/IN2P3, \\ UMR5822 IPNL, F-69622 Villeurbanne Cedex, France \\ A. M. Iyer \\ INFN-Sezione di Napoli, Via Cintia, 80126 Napoli, Italia
}

(Received 22 December 2017; published 5 March 2018)

\begin{abstract}
We investigate the production of heavy colored scalars and vectors and their relevance at the LHC for the study of vectorlike quarks $(T)$. These colored states $(C)$ are present in a large number of extensions of the standard model, in particular, in composite models and in extradimensional models. Assuming that these bosonic states are heavier than the vectorlike quarks (VLQ), we consider their production through the process $p p \rightarrow C \rightarrow t T$. Large QCD production cross sections for $C$ enable us to probe heavier masses for the VLQ, thereby allowing us to put stronger limits on the vectorlike quarks which are produced in their decay chain. We adopt a universal analysis strategy by including leptons under the classification of "jets," thereby limiting the bias towards a specific combination of final state. We also study the possibility of disentangling these scenarios from supersymmetric extensions of the standard model by using simple discriminants based on jet multiplicity and missing energy. We demonstrate that a simple set of cuts is sufficient to disentangle the VLQ signal from the backgrounds. In models with a moderate B.R. $(C \rightarrow T t)$, the analysis enables one to get a hint of VLQ masses as heavy as $3 \mathrm{TeV}$.
\end{abstract}

DOI: $10.1103 /$ PhysRevD.97.055002

\section{INTRODUCTION}

The standard model (SM) and its mechanism for the electroweak symmetry breaking is a quite successful description of the observed data. However a more fundamental description of the electroweak and strong interactions is still missing and many possible avenues are open in this respect. Two popular possibilities for physics beyond the SM suggest considering the Higgs boson either as a fundamental scalar particle (as for example in supersymmetric extensions of the $\mathrm{SM}$ ) or as a composite particle (as in composite models). This second possibility is usually described at the effective Lagrangian level, but if a more fundamental theory in terms of constituent fermions is considered, the presence of other bound states together with the vectorlike quarks (VLQ) is typically unavoidable. The common assumption in effective composite Higgs models is that all these extra states are much heavier. This can be indeed the case; however simple models

\footnotetext{
*Also at Institut Universitaire de France, 103 boulevard SaintMichel, 75005 Paris, France.

${ }^{\dagger}$ Also at Department of Theoretical Physics, Tata Institute of Fundamental Research, Mumbai, India.

Published by the American Physical Society under the terms of the Creative Commons Attribution 4.0 International license. Further distribution of this work must maintain attribution to the author(s) and the published article's title, journal citation, and DOI. Funded by SCOAP .
}

implementing the composite idea at the level of constituent new fermions can also allow composite states of similar mass range as the VLQs. These new states can be even lighter if protected by a symmetry. Typically independent studies are conducted for investigating the discovery potential of either the colored bosons or the vectorlike quarks. The possibility of composite colored scalars has been discussed in various extensions of the SM like [1,2]. The colored (composite) states can also manifest as spin-1 states generally corresponding to the KK excitations of gluons in warped extradimensional models [3,4]. Collider studies for these colored vectors have been considered in [5-8]. In parallel, comprehensive studies for the search of the vectorlike top partners, in both the composite and Randall-Sundrum (RS)-like scenarios, have been performed in [9-29].

Recently, there have been efforts to consider a unified search strategy for the heavy bosons and the VLQ. Typically it is assumed that the bosons are heavier than the VLQ, such that they decay into a VLQ and SM quark (often third generation is assumed). In particular, VLQs stemming from heavier states and decaying to multitop final states were considered in [30]. The first search for a spin 1 decaying into a $T t$ final state was performed by ATLAS [31] at $13 \mathrm{TeV}$ with $2.6 \mathrm{fb}^{-1}$ of data. Phenomenological analysis for similar topologies was considered for a heavy scalar $H$ decaying into a $T t$, suggesting kinematic variables to uniquely identify the presence of a VLQ state [32]. 
The optimal channel for the discovery of the spin-1 colored states is typically into a dijet final state, where the jet may also correspond to top jet. Observation of resonances in the high mass regime of the colored spin- 1 state is expected primarily in this channel due to the large production cross section. In this work we consider a complementary strategy for the study of the colored state $C$ and their decay including VLQ states.

The phase space for the search of VLQ through such channels can be considerably extended if the VLQ is produced in the decay of a heavier state. The noncolored states suffer a substantial reduction in the production cross sections at high mass. The corresponding effect on the colored excitations is comparatively much less and presents an avenue to probe the deeper realms of the VLQ masses which would be difficult to explore from direct search channels. A strategy to this effect was suggested in [23,33] with a heavy gluon and a VLQ, discussing different simplified models and search strategies. In the following we focus on the possibility of new colored states heavier than the VLQs, in order to show how the LHC reach in the searches of VLQs can be extended. Depending on the model framework, the identity of the final state will be different. We present a unified search strategy by considering jets initiated even by leptons (and photons). The analysis is hence applicable across all kinds of models with a similar particle content. This in turn gives bounds which can be applied directly on the composite Higgs models which are considered in the literature.

The paper is organized as follows: in Sec. II we briefly discuss the effective Lagrangian formalism and the different cases which are discussed. In Sec. III we perform a simplified analysis at the LHC in order to show the potential of these searches and discuss the possibility to discriminate such a signal from background and other cases giving similar final states. In Sec. IV we give our conclusions. A short appendix contains the details of the numerical simulation.

\section{EFFECTIVE LAGRANGIAN DESCRIPTION}

Fundamental composite models typically contain a large number of resonances: scalars, fermions and spin-1 particles. We discuss the effective Lagrangian description following two scenarios characterised by the nature of the particle content.

Case A: Heavy scalar and the fermionic VLQs.

To begin with, we consider the heavy scalar "mesons" and the fermionic "baryon" VLQs [34]. The colored pseudo-Nambu-Goldstone bosons (pNGB) are expected to have a mass which can be in the same range as the top partners; therefore their phenomenology crucially depends on the mass hierarchy. We assume here that the VLQs are lighter, therefore allowing their production in the decay chain of the colored bosons. As an example in the model considered in [2], the spectrum contains a complex color sextet (with charge $Q=4 / 3$ ) and a real color octet. The presence of such states is a generic prediction of any dynamics that also generates the VLQs.

The couplings of the sextet and octet can be written considering the invariance under color and charge at the effective Lagrangian level. The octet can only couple to a quark-antiquark pair. A sextet on the contrary can only be obtained by combining two quarks, as for the SU(3) representations $\mathbf{3} \otimes \mathbf{3} \supset \mathbf{6}$, or four antiquarks $\overline{\mathbf{3}} \otimes \overline{\mathbf{3}} \otimes$ $\overline{\mathbf{3}} \otimes \overline{\mathbf{3}} \supset \mathbf{6}$.

The effective Lagrangian after electroweak symmetry breaking is given as [2]

$$
\begin{aligned}
\mathcal{L}_{\text {eff }}= & \left|D_{\mu} \pi_{6}\right|^{2}-m_{\pi_{6}}^{2}\left|\pi_{6}\right|^{2}+\frac{1}{2}\left(D_{\mu} \pi_{8}\right)^{2}-\frac{1}{2} m_{\pi_{8}}^{2}\left(\pi_{8}\right)^{2} \\
& -V_{\text {scalar }}\left(\pi_{6}, \pi_{8}\right)+b_{R} \pi_{6} t_{R}^{c} t_{R}^{c}+b_{L} \pi_{6}^{c} t_{L} t_{L} \\
& +c \pi_{8} t_{R}^{c} t_{L}+\text { H.c. }
\end{aligned}
$$

where $t_{L / R}$ are chiral Weyl spinors $\left({ }^{c}\right.$ indicates the charge conjugation) and $V_{\text {scalar }}$ is a generic self-interaction between the scalars. Parity is not conserved in (1), because only the coupling $b_{R}$ corresponds to gauge invariant operators. The other couplings are generated only at the electroweak symmetry breaking.

Note that we couple the sextet only to the third generation quarks, as is typically assumed in composite models based on partial compositeness considerations, but, in principle, a coupling to the other quark generations is also possible.

Case B: Heavy vector and the fermionic VLQs:

This is typically characteristic of spectrum in warped extradimension models. For simplicity we consider the VLQ to be a singlet under SU(2). However, as is described later, the analysis proceeds independently of any specific final state and is fairly robust. This implies the analysis is relevant for any new physics (NP) topology which has lead to three particle multiplicity in the final state (these final states are identified as jets in Sec. III). The effective Lagrangian for particle content under consideration is given as

$$
\begin{aligned}
\mathcal{L}_{R S} \supset & \bar{q} T^{a} \gamma^{\mu} q G_{\mu}^{a}+\left(Y_{t} \bar{Q}_{3} H t_{p}+M_{t^{\prime}} \bar{t}_{p} t_{p}\right. \\
& \left.+Y_{t} \bar{Q}_{3} H t+\text { H.c. }\right) .
\end{aligned}
$$

Here $G_{\mu}$ represents the first KK excitation of the gluon in RS-like models and $H$ is the SM Higgs doublet. For the specific case under consideration, the vectorlike state $t_{p}$ mixes with the top quark leading to two eigenstates $\left(t_{1}, t_{2}\right)$, where $t_{1}$ is identified as the SM top and $t_{2}$ is the top partner. Since it is a singlet $\mathrm{SU}(2), t_{2}$ has only three decay modes: $W b, Z h$, th with the $W b$ constituting $50 \%$ of the total branching fraction. 


\section{A. Spin-1, sextet and octet masses}

The mass terms for the vectors, colored sextet and octet mesons were described in detail in [2] in the case of the global symmetries $S U(4)$ and $S U(6)$ for the fundamental fermions giving rise to the VLQ baryons, the various pNGB "pions" and the spin-1 vectors. The spin-1 states are allowed to have an $S U(6)$ invariant mass term which is typically a heavy mass scale. The $S U(6)$ symmetry is also broken explicitly by a mass term, which gives the mass ballpark for the colored pNGBs. For the VLQs an extra mass term is also present; therefore the naive expectation is that the mass of the VLQs will receive both a contribution from the dynamical and explicit $S U(6)$ breaking, while the colored pNGBs will only receive a contribution from the explicit breaking. The composite spin-1 resonances, due to the $S U(6)$ invariant mass term, are typically expected to be heavy.

This picture can be modified when the model is close to the conformal window, where large anomalous dimensions may be generated for some of the composite operators. In the following we consider only the effective model for the bound states and treat the mass terms as parameters of the model, without referring to a particular fundamental dynamics behind them (which is anyway model dependent and which requires detailed lattice simulations in order to establish the spectrum of the low energy theory). The situation we examine is the one in which the colored sextet and octet mesons as well as the spin-1 resonances are heavier than the VLQ top partners, allowing the decay modes of the $\pi_{6}, \pi_{8}$ and spin-1 resonances to the VLQ top partners.

\section{B. Pair vs single production of heavy colored bosons and LHC bounds}

The possibility of new massive color octet vector bosons with sizable decay into the VLQs was considered by [35-39] and the impact on the LHC searches was assessed using as an example a simplified version of the composite Higgs model, based on the $S O(5) / S O(4)$ coset and a composite right-handed top quark $[16,40]$. The focus in [39] was on pair production of the VLQs. The present limits on VLQ masses are however higher and single production of the VLQs, even if more model dependent, becomes more constraining as the single production cross section becomes larger than pair production for large enough masses of the VLQs. In the following we therefore focus on single production of the VLQs in the decay chain of colored vector bosons, and sextet and octet colored mesons.

The present limits on VLQs come from the detailed searches of their decay products (both for pairs and single production) assuming either a $100 \%$ branching in one specific decay mode or a set of decay modes (typically those into a SM quark plus the Higgs boson or the W, Z gauge bosons). The lower bounds for the masses are typically in the $\mathrm{TeV}$ mass range. We use them as a guideline, but lower mass values can be possible if extra decay modes are present or dominant. For example top partners with masses below $900 \mathrm{GeV}$ are excluded independently of whether they decay into $\mathrm{Zt}, \mathrm{Ht}, \mathrm{Wb}$ or $\mathrm{St}$ (where $S$ gives either missing energy or $b$ quarks) or any combinations of those [41].

Experimental searches using the VLQ single production from heavy colored gluon were recently performed in the four $b$-quarks final state [42]. In that case stringent limits on the production cross section times branching ratio can be set. This kind of analysis can be generalized to other final states and to different heavy colored objects. A similar analysis involving colorless $Z^{\prime}$ decaying into a $T \bar{t}$ was considered in [31] in a multijet final state. Indeed, while color octets are typically considered, color sextets are not. In the following we consider those heavy states (including the sextet) as a mechanism to produce or bound vectorlike top partners $T$ in association with a SM top quark.

\section{COLLIDER ANALYSIS AT THE LHC}

In this section we explain the details of the collider strategy employed in our analysis. Though there are two possibilities for the spin and the representation of the colored objects, the analysis proceeds in a democratic fashion without any bias on the nature of the coupling. For simplicity they are collectively referred to as $C$. As explained earlier, large production cross sections for the colored states not only enable us to probe heavier masses for such states, but also serve as a portal to the existence of heavy vectorlike states. We consider the production of the these states (as a typical feature of the composite models we consider a top partner $T$ ) in association with a SM top through the following process:

$$
p p \rightarrow C \rightarrow t T .
$$

The electric charge of $T$ is the same as that of the SM top (top partners); however, depending on the representation of $T$ under $(S U(3), S U(2))$, the final states are different. The following two cases are discussed:

(i) Case $\mathrm{A}-(\overline{3}, 1)$ : They are produced in association with tops of the same sign through colored objects which transform as a sextet. The only possible decay mode is $T \rightarrow t g$.

(ii) Case B-(3,1): They are produced in association with opposite sign tops though colored octets. The dominant decay mode is $T \rightarrow W b$, which accounts for $50 \%$ of the branching fraction. The subdominant modes include $Z t$ and $t h$, each of which accounts for $25 \%$.

Though the final states in the above two scenarios are different, they are characterized by the presence of three four vectors at the particle level. Typically in a collider, final states are characterized by combinations of jets, 
leptons, photons and missing energy. Heavier objects like $W, Z, t$ are reconstructed from their final state decay products. With increasing boost of the particles, decay products of objects like $t, W, Z$ are concentrated in a small annular region typically of radius $\Delta R \sim 2 \mathrm{~m} / p_{T}$, where $m$ is the mass of the corresponding object having transverse momentum $p_{T}$. As a result these objects also fall into the broad classification of jets and can then be considered on the same footing as the jet initiated by a gluon or a light quark to begin with. In order to put this into practice, it is essential that the size of the jet-reconstruction radius is such that the decay products of the heavier objects (especially the top) are captured and depend on the transverse momentum. As an illustration we consider the following two benchmark points:

$$
\begin{aligned}
& \mathrm{BP} 1: m_{C}=3 \mathrm{TeV} m_{T}=1.7 \mathrm{TeV} \\
& \text { BP2: } m_{C}=3.5 \mathrm{TeV} m_{T}=2.5 \mathrm{TeV} .
\end{aligned}
$$

Figure 1 gives the parton level $p_{T}$ for the two benchmark points originating from either the $\mathrm{C}$ or the $\mathrm{T}$. The plots in the right column correspond to the $t$ tg final state (case A) and left column correspond to the $t W b$ final state (case B).
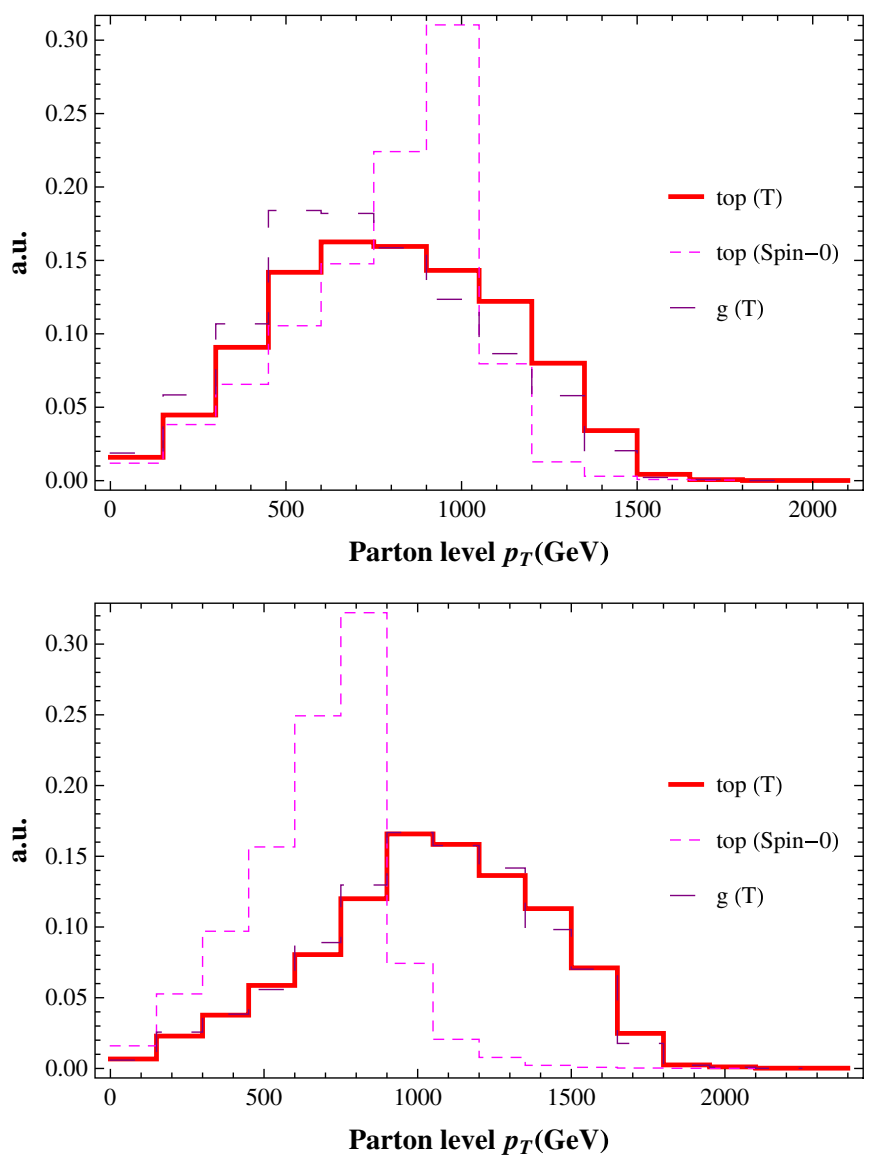

It can be clearly seen that a choice of $R=0.8$ for forming these jets is sufficient to capture all the information from the heavier objects. The Appendix gives the details of the simulation employed in our analysis.

\section{A. Signal discrimination}

In view of identifying a unique signature for the presence of VLQs, it is essential to identify different processes which may impersonate the signal. These include both SM and other NP processes and are discussed below.

QCD: Since the final state is associated with three hard partons which eventually form jets, we require the presence of at least three jets in the event. The analysis with lepton jets makes it necessary to find a strategy to tackle the QCD background. We adapt the following event selection criteria: Only events which have a single isolated lepton are accepted. This is equivalent to the condition that the lepton subjet inside the fat jet satisfies the isolation criteria. This is clearly illustrated in Fig. 2, which computes the angular separation $\left(\Delta R_{j_{i}}\right)$ between the conventional isolated leptons and the eflow jets. We compute $\Delta R_{l j_{i}}$ for the three leading jets in the event, i.e. $i=1,2,3$, and plot the minimum. The small smearing outside the jet radius
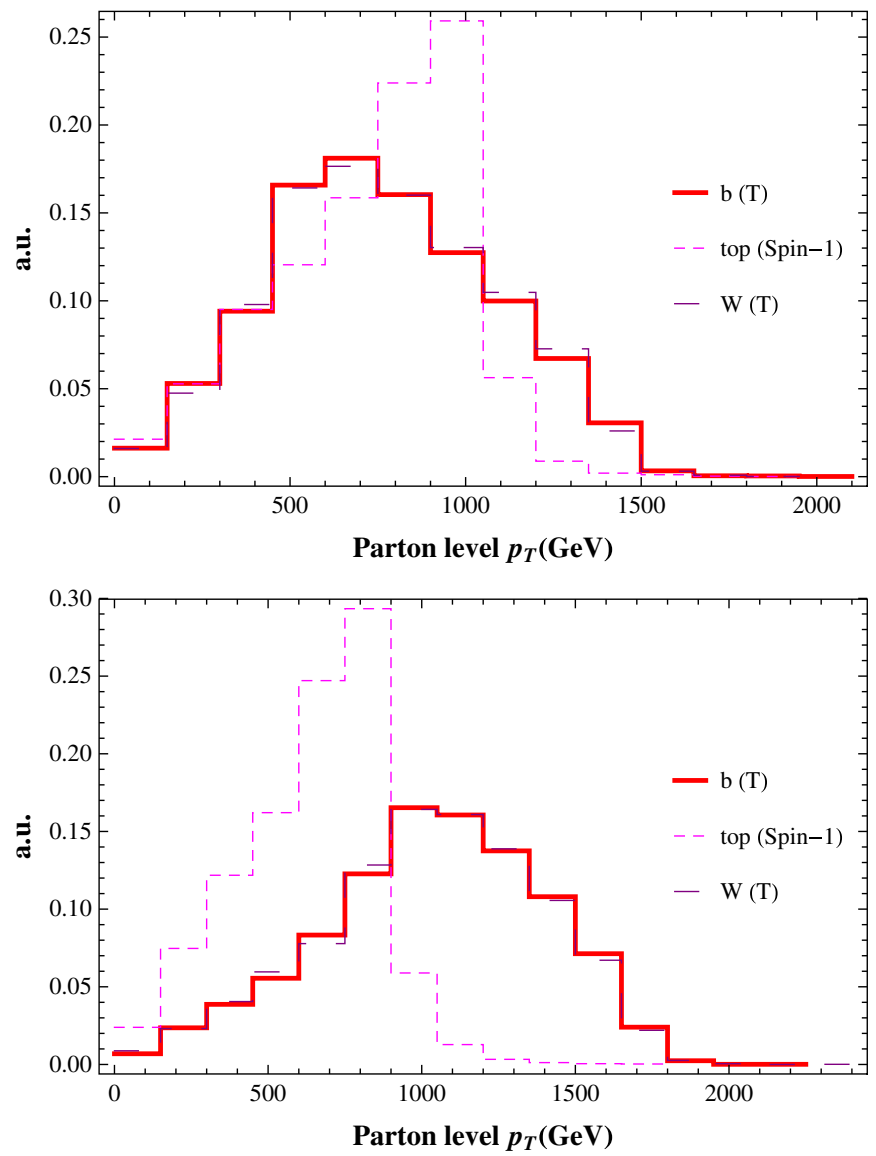

FIG. 1. The left column gives the parton level $p_{T}$ for the $t t g$ final state while the right column gives the corresponding $p_{T}$ for the $t W b$ final state. The top row corresponds to the BP1 while the bottom row corresponds to BP2. 


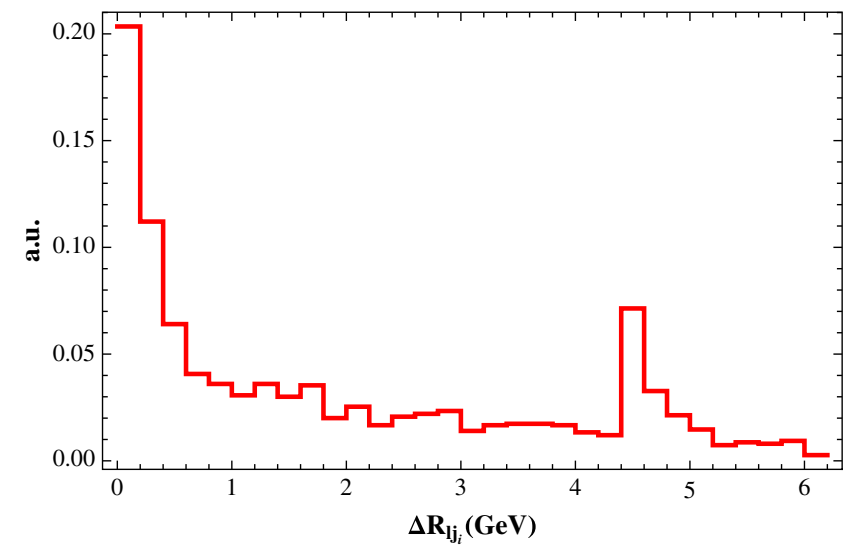

FIG. 2. Angular distance between the isolated leptons and the jets constructed using eflow four vectors.

$R=0.8$ corresponds to events with larger jet multiplicities or with significantly softer leptons which do not originate from the signal topology. It is to be noted that since such topologies are always associated with at least a top in the final state, the isolated lepton generally corresponds to the leptonic decay of the top. These criteria are only used to filter events and the isolated lepton is not used further in the analysis. For the events which pass this criteria, we take the eflow four vectors and cluster them into jets. Note that eflow four vectors comprise track, photons and neutral hadrons. The resulting jets also include leptons in addition to the conventional hadrons. After accounting for the energy smearing of the jets, they are further used in the analysis. The advantage of dealing with these "universal" jets as opposed to the conventional jets + leptons has the main advantage of being democratic across different possibilities of final state. Though it may seem that the presence of a single isolated lepton may make the analysis less generic, it must be noted that the final state is always associated with the presence of a $t$ : either from the VLQ or from the vertex of the decay of the colored boson. As a result the lepton in this case can be attributed to the top. The relaxing of these criteria would warrant the investigation of the substructure of these jets $[43,44]$ to distinguish the signal. For instance, using the eflow jets, we can look for the lepton subjet inside the jet and study the following substructure variables:

(A) Track: A lepton jet is likely to be associated with a single track as it is a useful discriminator against a QCD jet which is associated with the large track multiplicity $[43,45,46]$.

(B) Hadronic energy content inside the subjet: Further one can use the cut on the following substructure variable,

$$
\theta_{J} \equiv \frac{1}{E_{J}} \sum_{i \in\{\mathrm{J}, \mathrm{HCAL}\}} E_{i} \quad \text { where } E_{J} \equiv \sum_{i \in J} E_{i}
$$

which is a measure of the energy deposited by the jet in the $\mathrm{H}$-cal to the total energy of the jet. For lepton subjets, $\log \left[\theta_{J}\right] \ll 0$ while for QCD $\log \left[\theta_{J}\right] \sim-0$. 4 . It was shown that a combination of track and $\log \left[\theta_{J}\right]$ can reduce the QCD to less than 1\% [43].

$t \bar{t}+$ jets: In accordance with the discussion above and event selection criteria explained in the Appendix, $t \bar{t}+$ jets constitutes one of the most dominant SM backgrounds. The matrix element for the process is given as

$$
\begin{aligned}
\mathcal{M}_{t \bar{t}+\mathrm{jets}}= & \mathcal{M}(p p \rightarrow t \bar{t})+\mathcal{M}(p p \rightarrow t \bar{t}+j) \\
& +\mathcal{M}(p p \rightarrow t \bar{t}+j+j),
\end{aligned}
$$

where $j$ is a parton. As seen in Fig. 1, the signal kinematics is accompanied with high $p_{T}$ partons. In order to populate the phase space in the signal regime, the events are simulated by requiring the minimum invariant $p_{T}$ to be $800 \mathrm{GeV}$. The left panel of Fig. 3 gives a comparison of $p_{T}$ of the leading jet for the background and the two benchmark points. Though BP1 and BP2 have been plotted for the $t$ tg final state, $t W b$ or any final state from similar NP masses also exhibits the same behavior. Since all the three plots have a similar pattern, they suggest that the

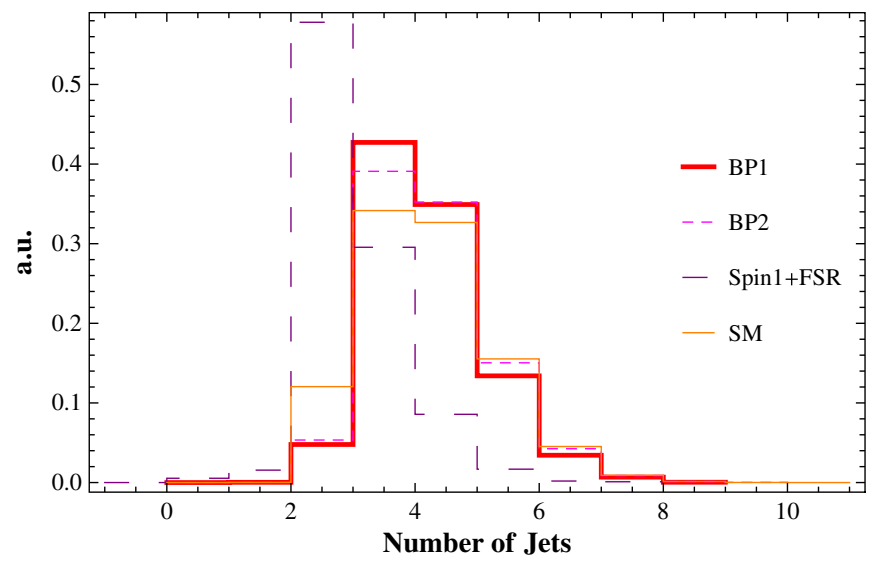

FIG. 3. $p_{T}$ of the leading jet for the signal and two benchmark points. 

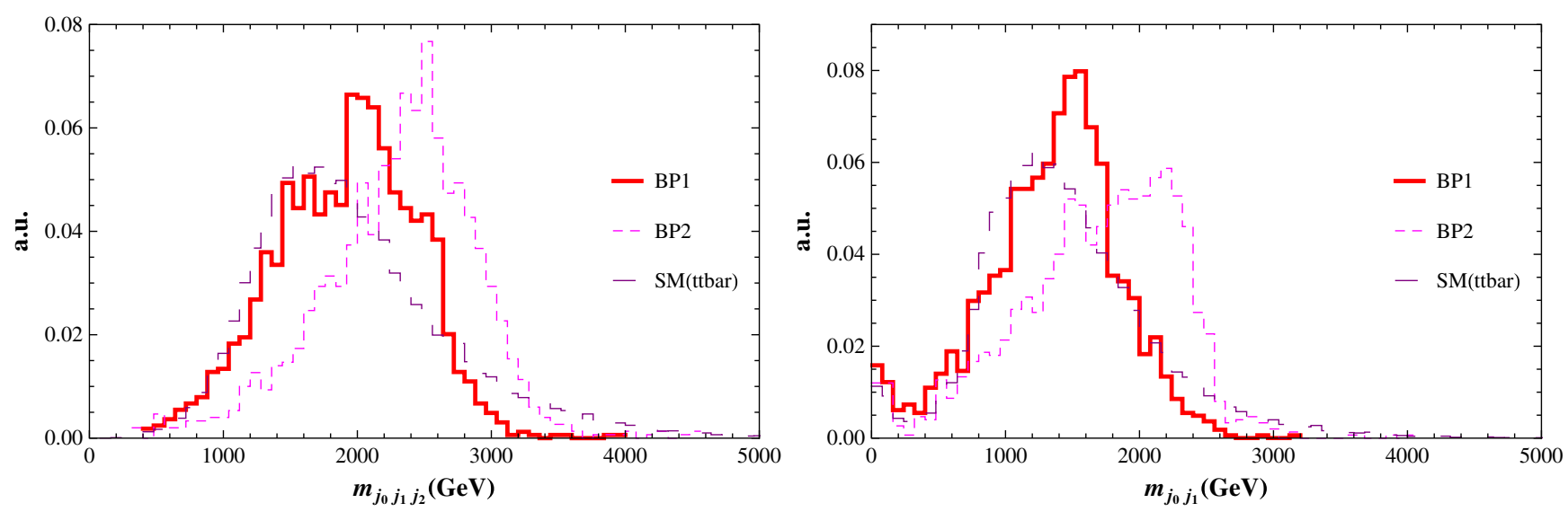

FIG. 4. The total invariant mass of the three leading jets (left) and the invariant mass of the two leading jets (right).

background has been fairly well represented in the signal phase space. The effective cross section is reduced to $1740 \mathrm{fb}$ and we simulate 0.5 million events for the same. This background can be limited by cuts on the $p_{T}$ of the leading jets as well as the invariant mass of the three leading jet.

$W+$ jets: The matrix element for $W+$ jets with $W$ decaying leptonically is given as

$$
\begin{aligned}
\mathcal{M}_{W+\mathrm{jets}}= & \mathcal{M}(p p \rightarrow W)+\mathcal{M}(p p \rightarrow W+j) \\
& +\mathcal{M}(p p \rightarrow W+j+j) .
\end{aligned}
$$

Similar to the $t \bar{t}+$ jets, the events are simulated by requiring the minimum invariant $p_{T}>800 \mathrm{GeV}$. Though the cross section for this process is higher than that of $t \bar{t}+$ jets, the requirement of the leading jet having $p_{T}>800$ and the invariant mass makes the effective cross section much smaller as is shown in Table I.

$Z+$ jets: The matrix elements for the process are

$$
\begin{aligned}
\mathcal{M}_{Z+\mathrm{jets}}= & \mathcal{M}(p p \rightarrow Z)+\mathcal{M}(p p \rightarrow Z+j) \\
& +\mathcal{M}(p p \rightarrow Z+j+j) .
\end{aligned}
$$

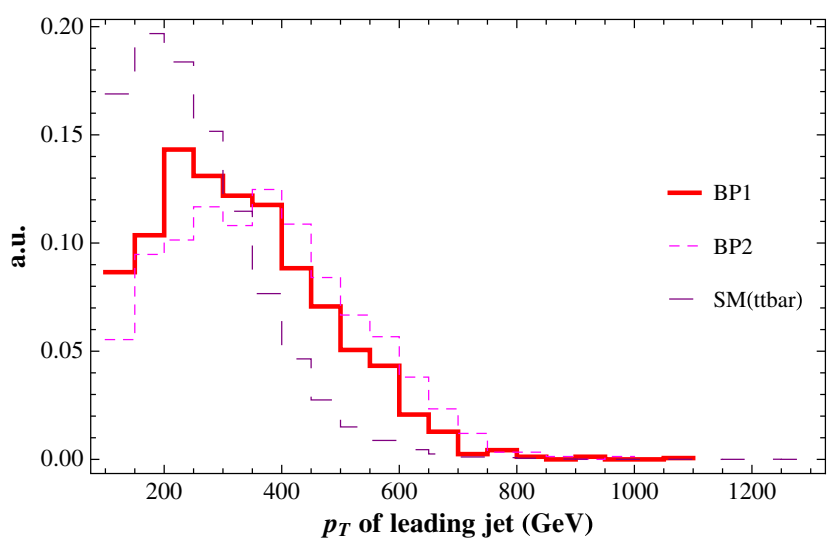

FIG. 5. $p_{T}$ distribution for the third leading jet.
The raw cross section for this process is an order of magnitude less than $W+$ jets. In the high $p_{T}$ regime as is being probed here, the $Z$ is likely to be boosted and the leptons from the $Z$ are likely to be collimated. Thus it is highly likely that the leptons inside the $Z$ jet either result in two isolated lepton subjets or none of them pass the isolation criteria. The latter effect was observed for the case of collimated photons in [47]. Thus, this SM can be further depleted by the requirement of a single isolated lepton in the jet and the three jet criteria.

$C \rightarrow t \bar{t}$ : In the presence of a heavy colored object, the $t \bar{t}$ final state constitutes its most favorable decay mode and must be accounted for. We generate these events by also considering the emission of a parton from the final state top. The total matrix element for the process is given as

$\mathcal{M}_{C=t t}=\mathcal{M}(p p \rightarrow C \rightarrow t \bar{t})+\mathcal{M}(p p \rightarrow C \rightarrow t \bar{t}+j)$.

Supersymmetry (SUSY) processes: We stress here that SUSY is not a background but a different type of possible signal which should be discriminated from the case under study. It is essential to pin down the topology originating from the colored boson and a VLQ. Minimal supersymmetric extensions of the SM do not contain vectorlike states. However certain decays of colored objects like the stops or the gluinos may potentially impersonate the VLQ signal, either in terms of jet multiplicity or the number of isolated leptons. A similar study to this effect was considered in [38] for the decay of heavy gluon to VLQ. In general there are a plethora of processes in the minimal supersymmetric standard model which may exhibit similar kinematics or multiplicity of certain combinations of final state collider objects. As a representative, we consider the following two processes:

(a) Pair production of stops: We consider the pair production of $1 \mathrm{TeV}$ stops with the decay $\tilde{t} \rightarrow t \chi_{1}^{0}$. The 
final state in this case is $t t+E_{T}^{\text {miss }}$. The visible final states in this case are similar to the SM $t \bar{t}+$ jets and the $C \rightarrow t \bar{t}$.

(b) Pair production of gluinos: Searches for the gluinos in the $1 l+$ jets $+E_{T}^{\text {miss }}$ final state restrict the mass of the gluinos to be $m_{\tilde{g}} \gtrsim 2 \mathrm{TeV}$.

All these processes are now collectively referred to as background to our spin-1 + VLQ decay topology. We now list the different variables which are useful in distinguishing the VLQ signal from these background processes.

(i) Jet multiplicity $\left(n_{J}\right)$ : The signal is characterized by a peak at 3 in the jet distribution as shown in right panel of Fig. 3. The other NP processes are however characterized by smaller $(C \rightarrow t \bar{t}$ or $\bar{t} \bar{t})$ or larger $(\tilde{g} \tilde{g})$ jet multiplicities. While the SM process exhibits a similar nature, it can be substantially reduced by a minimum requirement on the leading jet $p_{T}$.

(ii) $p_{T}$ of the leading jet $\left(p_{T}^{(0)}\right)$ : For both the benchmark points in Eq. (4), the most energetic partons originate from the spin-1 vertex and are likely to constitute the leading jet. Figure 3 (left panel) gives the $p_{T}$ distribution for the background process (left) and the signal (right). One can expect the $C \rightarrow t \bar{t}$ to have a similar distribution. However, this is useful in eliminating the SM and the SUSY background.

(iii) Reconstruction of the $W$ boson mass: For the topology under consideration we demand that the missing momenta is only due to the neutrino. The $z$ component of the neutrino is explicitly evaluated by assuming that it originates from the $W$. This is particularly useful in keeping SUSY backgrounds at bay.

(iv) Total invariant mass $m_{j_{0} j_{1} j_{2}}$ : Owing to the presence of missing energy from the neutrino, the total invariant mass of the three leading jets is likely to exhibit an edge at the mass of the spin-1 resonance. Left plot of Fig. 4 gives the distribution for this variable for the SM background and the two benchmark points. It is clear that as the mass of the colored resonance increases, the discriminatory capability of this variable becomes even stronger. (v) $p_{T}$ of the third leading jet $\left(p_{T}^{(2)}\right)$ : The partons from $T$ are also considerably energetic, result in the corresponding jets with large $p_{T}$ and generally constitute the subleading jets. Figure 5 gives the distribution of the $p_{T}^{2}$ for the third leading jet for both the background and the signal. Though this cut is not used in the results, it presents a possibility for an additional variable to be used in a multivariate analysis using boosted decision tree [48].

\section{B. Results and discussions}

Table I gives the background and signal efficiencies at each level of the cuts imposed. Note that the $t W b$ final state corresponds to a RS-like scenario with singlet VLQ $T$. Though the efficiencies are quoted only for a particular final state for simplicity, the robustness of the analyses ensures that one can expect similar efficiencies for the other final state, viz., $t t h, t t z$. Thus in a realistic scenario all possible decay modes of the VLQ are taken into account without any particular bias.

The large $p_{T}$ cut $\left(p_{T}>800 \mathrm{GeV}\right)$ is imposed to ensure that the final state jets have originated from a massive resonance. This is further complemented by the requirement of a minimum on the invariant mass of the three leading jets $\left(m_{j_{0} j_{1} j_{2}}>2000 \mathrm{GeV}\right)$. At this point it is important to note that these cuts are also very useful in keeping the SUSY backgrounds in check. Note that SUSY particles are typically produced in pairs and for final states from SUSY topologies to pass these basic cuts, it requires the pair production of very massive SUSY particles. Subsequently, this results in a significant drop in their production cross section, much beyond the range of luminosities discussed here.

Irrespective of the final state, both signal topologies exhibit similar efficiencies, thus validating our analysis. The efficiencies for BP2 are likely to be slightly higher as the new physics states are more massive, thereby resulting in more events passing the $p_{T}$ criteria. We now discuss the luminosity reach corresponding to the efficiencies quoted in Table I. For brevity we discuss the numbers corresponding to the $t$ tg final state.

TABLE I. Signal and background efficiencies for different levels of cuts employed. The isolated lepton in the parenthesis refers to the criteria where the lepton inside the fat jet satisfies the isolation criteria.

\begin{tabular}{|c|c|c|c|c|c|c|c|c|}
\hline \multicolumn{5}{|c|}{$n_{J}>=3$ (isolated lepton) } & \multirow{2}{*}{\multicolumn{2}{|c|}{ Signal(BP1) }} & \multirow{2}{*}{\multicolumn{2}{|c|}{ Signal(BP2) }} \\
\hline \multirow[b]{2}{*}{ Cuts } & \multicolumn{4}{|c|}{ Background samples } & & & & \\
\hline & $t \bar{t}+$ jets & $W+$ jets & $Z+$ jets & $C \rightarrow t \bar{t}$ & $\operatorname{ttg}$ & $t W b$ & $\operatorname{ttg}$ & $t W b$ \\
\hline$\ldots$ & 0.18 & 0.32 & 0.24 & 0.053 & 0.16 & 0.23 & 0.15 & 0.23 \\
\hline$p_{T}^{(0)}>800 \mathrm{GeV}$ & 0.02 & 0.011 & 0.004 & 0.007 & 0.027 & 0.026 & 0.033 & 0.029 \\
\hline$m_{j_{0} j_{1} j_{2}}>2000 \mathrm{GeV}$ & 0.013 & 0.005 & 0.003 & 0.0027 & 0.014 & 0.013 & 0.027 & 0.017 \\
\hline
\end{tabular}


For BP1, the typical production cross section for the colored boson is $100 \mathrm{fb}^{-1}$. Furthermore the B. R. of the colored boson $(C \rightarrow t T)$ depends on the model. The authors of [23] considered different possibilities when the colored boson is a heavy gluon. Assuming a $60 \%$ branching fraction these channels can be probed at a $S / \sqrt{B} \sim 5 \sigma$ sensitivity with $800 \mathrm{fb}^{-1}$ of data. Extending the luminosity to $3000 \mathrm{fb}^{-1}$ even models with $\sim 30 \%$ branching ratios can be probed at a similar sensitivity.

For BP2 the associated production cross sections are much lower with typical values $\sim 30 \mathrm{fb}$ [8]. As a result, with $60 \% \mathrm{~B}$. R. of the colored state into the $t T$ pair, $2400 \mathrm{fb}^{-1}$ of data is required for $S / \sqrt{B} \sim 5 \sigma$ sensitivity. In this case B. R. as low as $54 \%$ can be probed with $3000 \mathrm{fb}^{-1}$ of data.

We also comment on the case where the mass of VLQ for $\mathrm{BP} 2$ is increased to $3 \mathrm{TeV}$. In this case the efficiencies are similar to that for BP2 in Table I. B. R. in this case are however likely to be smaller owing to the smaller mass difference: $\Delta m=m_{C}-m_{T}$. Smaller B. R. of up to $33 \%$ can be probed with $3000 \mathrm{fb}^{-1}$ at $3 \sigma$ sensitivity thereby making a strong case for future colliders.

Mass of the vectorlike quark: While the simple cuts in Table I help us in identifying the specific topology under consideration, it is also worthwhile to get a rough estimate of the mass of the VLQ. Since we are looking at the complete jet final state, the identity of the partons from the VLQ is not straightforward and hence one must consider different combinations of the invariant mass distributions. The right panel of Fig. 4 gives the invariant mass of the two leading jets $m_{j_{0} j_{1}}$ and for both the benchmark points the distribution terminates at roughly the mass of the VLQ. While this is useful, it can only be used as a guideline. In practice one must consider other combinations to help in uniquely determining the mass.

At this instance we compare our analysis with the authors of [36], who were the first to propose this topology. The analysis in the paper proceeds by considering a Wtb final state with the requirement of a single isolated lepton and a minimum of three jets in the final state. The jets are reconstructed with $R=0.4$ corresponding to the standard reconstruction radius used in search for NP. In addition there is a specific requirement of two $b$ tags in the final state. The final state in this case is likely to be characterized by a relatively larger jet multiplicity and is likely to resemble that of $G \rightarrow \bar{t} \bar{t}$. In the parameter space where $G \rightarrow \bar{t} t$ branching fraction is low this strategy is extremely effective for the particular final state under consideration. However the analysis proposed in the paper proceeds in a manner allowing us to be agnostic about the VLQ decay mode by considering fat jets with $R=0.8$. Thus all heavy light topologies are characterized primarily by a three jet multiplicity. This is crucial to our analysis as we are keen on looking for an exclusive signal for the presence of the VLQ. As illustrated in Fig. 2, jet multiplicity is central to distinguishing it from the $G \rightarrow t \bar{t}$ decay mode especially in regions of parameter space where the $t \bar{t}$ decay mode is prominent.

The analysis proposed in this work may serve as a discovery mode for an exclusive hint for the presence of the VLQ which are produced from the decay of a heavy colored boson. It is to be noted that the presence of an isolated lepton (or lepton subjet) in this work corresponds to the requirement of an isolated lepton in [36]. Thus, in case of a discovery, one can reanalyze the events by adapting the strategy proposed in [36] to get a further hint of the particular decay mode and to further enhance the signal sensitivity over the background.

\section{CONCLUSIONS}

Colored bosons are present in typical extensions of the SM and, in particular, in fundamental composite models and extradimensional models. These models also typically contain VLQ states. We have investigated the possibility of producing VLQs in association with those colored states using a generic effective Lagrangian framework taking into account the possible couplings based on the quantum numbers of those particles. In this respect the analysis performed here can be therefore used for various different models. For the collider analysis at the LHC we have shown that a common strategy can be used for these resonances. Furthermore we focus our study to final states which are not specific and that can be broadly classified as jets, without searching for specific detailed signatures (for example containing leptons or reconstructing gauge boson masses). While this approach is typically less constraining for searches and establishing bounds, it is however quite robust and generic, allowing in this simplified study to used previous analyses and to apply the results to a wide class of models. The main outcome of the present study is that bounds on VLQs can be improved beyond the reach of standard searches if the production in association with heavy colored bosons is considered. This reach can be extended to $\sim 3 \mathrm{TeV}$ in cases with heavy colored state $m_{c} \sim 3.5-3.7 \mathrm{TeV}$ having a moderate to large B.R. $(C \rightarrow t T)$. The hypothesis of extra colored states is motivated by their presence in a large number of models and is by no means an exception. The presence of a color octet is considered in some of the existing searches, for example in the form of a heavy gluon, but more generally other quantum numbers are possible for these states (for example, the sextet) and their phenomenology and implications are not fully explored. Their study in conjunction with VLQs was therefore one of the main motivations for the present study. Finally in the case that such events are seen at the LHC, we briefly address the possibility of discriminating such a scenario from other models and, in particular, supersymmetric models which are characterized by the absence of VLQs. However the decay of colored objects like stops or gluinos may produce, in principle, VLQ-like signals, 
either in terms of jet multiplicity or the number of isolated leptons. We have shown that jet multiplicity is different in those cases and also that other discriminating criteria can be used, such as the study of missing energy and the edges of distributions. The analysis can be further improved by considering using multivariate techniques, thus providing better perspectives on the existence of these states.

\section{ACKNOWLEDGMENTS}

We acknowledge many useful discussions with Ushoshi Maitra and her collaboration in the initial stages of the project. A. D. is partially supported by the "Institut Universitaire de France," the Lyon Institute of Origins (Labex-LIO) under Grant No. ANR-10-LABX-66 and Fédération de Recherche "André Marie Ampère" (FRAMA, Grant No. FR3127). A. M. I. was supported in part by MIUR under Grant No. 2015P5SBHT and by the INFN research initiative ENP. A.M. I. acknowledges the hospitality at IPN Lyon where significant parts of the project were discussed. We also acknowledge the organizers of From Strings to LHC IV where parts of the project were discussed. A. D. and A. M. I. also thank organizers of Les Houches Workshop series 2017 where part of the discussions were held. We acknowledge the computational facilities at the Department of Theoretical Physics, TIFR. We warmly acknowledge the support of the CNRS Laboratoire International Associé (LIA) Theoretical High Energy Physics (THEP) and the IndoFrench Network on High Energy Physics (INFreHEPNET) of the Indo-French Centre for the Promotion of Advanced Research (CEFIPRA/IFCPAR).

\section{APPENDIX: DETAILS OF THE SIMULATION}

In this section we outline the details of the simulation and jet-clustering algorithm employed in our analysis.

(i) The events are generated using MADGRAPH [49] at $13 \mathrm{TeV} \mathrm{CM}$ energy using parton distribution functions NNLO1. The model file for the processes is generated using FEYNRULES [50]. The events are then showered and hadronized using PYTHIA 8 [51] and then passed to the DELPHES 3.3.2 [52] detector simulator using the CMS card.

(ii) The jets are clustered with the particle-flow objects using FASTJET [53] with the anti-kt [54] with the following parameters: $R=0.8$ and $p_{T}^{\min }=$ $100 \mathrm{GeV}$.

(iii) The extracted jets used for the analysis are not subject to the unique object finder module to distinguish it from the isolated leptons. This implies that the jets now include even those initiated by the leptons.

(iv) Jet matching becomes necessary only when generating the matrix elements with the soft partons in MADGRAPH and correspondingly needs to be mapped to the partons produced by PYTHIA during showering. However showering does not produce high $p_{T}$ partons as required by the signal and in the corresponding $p_{T}$ regime for the background and hence it does not alter jet multiplicity or the cross section.

(v) Requirement of a single isolated lepton is only imposed at the event selection criteria to reject the QCD background. The standard parameters for lepton isolation are chosen: isolation radius of $R=$ 0.4 and the $p_{T}$ fraction of the activity around lepton candidate to be less than 0.1 of the $p_{T}$ of the lepton.
[1] R. Lewis, C. Pica, and F. Sannino, Phys. Rev. D 85, 014504 (2012).

[2] G. Cacciapaglia, H. Cai, A. Deandrea, T. Flacke, S. J. Lee, and A. Parolini, J. High Energy Phys. 11 (2015) 201.

[3] L. Randall and R. Sundrum, Phys. Rev. Lett. 83, 3370 (1999).

[4] T. Gherghetta and A. Pomarol, Nucl. Phys. B586, 141 (2000).

[5] K. Agashe, A. Belyaev, T. Krupovnickas, G. Perez, and J. Virzi, Phys. Rev. D 77, 015003 (2008).

[6] B. Lillie, L. Randall, and L.-T. Wang, J. High Energy Phys. 09 (2007) 074.

[7] M. Guchait, F. Mahmoudi, and K. Sridhar, Phys. Lett. B 666, 347 (2008).

[8] A. M. Iyer, F. Mahmoudi, N. Manglani, and K. Sridhar, Phys. Lett. B 759, 342 (2016).
[9] T. Han, H. E. Logan, B. McElrath, and L.-T. Wang, Phys. Rev. D 67, 095004 (2003).

[10] R. Contino, L. Da Rold, and A. Pomarol, Phys. Rev. D 75, 055014 (2007).

[11] M. Carena, J. Hubisz, M. Perelstein, and P. Verdier, Phys. Rev. D 75, 091701 (2007).

[12] S. Matsumoto, T. Moroi, and K. Tobe, Phys. Rev. D 78, 055018 (2008).

[13] C. Anastasiou, E. Furlan, and J. Santiago, Phys. Rev. D 79, 075003 (2009).

[14] G. D. Kribs, A. Martin, and T. S. Roy, Phys. Rev. D 84, 095024 (2011).

[15] S. Gopalakrishna, T. Mandal, S. Mitra, and R. Tibrewala, Phys. Rev. D 84, 055001 (2011).

[16] A. De Simone, O. Matsedonskyi, R. Rattazzi, and A. Wulzer, J. High Energy Phys. 04 (2013) 004.

[17] N. Vignaroli, J. High Energy Phys. 07 (2012) 158. 
[18] S. Gopalakrishna, T. Mandal, S. Mitra, and G. Moreau, J. High Energy Phys. 08 (2014) 079.

[19] M. Buchkremer, G. Cacciapaglia, A. Deandrea, and L. Panizzi, Nucl. Phys. B876, 376 (2013).

[20] A. Banfi, A. Martin, and V. Sanz, J. High Energy Phys. 08 (2014) 053.

[21] J. Li, D. Liu, and J. Shu, J. High Energy Phys. 11 (2013) 047.

[22] B. Gripaios, T. Mller, M. A. Parker, and D. Sutherland, J. High Energy Phys. 08 (2014) 171.

[23] M. Chala, J. Juknevich, G. Perez, and J. Santiago, J. High Energy Phys. 01 (2015) 092.

[24] M. Endo, K. Hamaguchi, K. Ishikawa, and M. Stoll, Phys. Rev. D 90, 055027 (2014).

[25] M. J. Dolan, J. L. Hewett, M. Krmer, and T. G. Rizzo, J. High Energy Phys. 07 (2016) 039.

[26] S. Dawson, I. M. Lewis, and M. Zeng, Phys. Rev. D 91, 074012 (2015).

[27] C.-Y. Chen, S. Dawson, and I. M. Lewis, Phys. Rev. D 90, 035016 (2014).

[28] S. Dawson, E. Furlan, and I. Lewis, Phys. Rev. D 87, 014007 (2013).

[29] D. Barducci and C. Delaunay, J. High Energy Phys. 02 (2016) 055.

[30] A. Deandrea and N. Deutschmann, J. High Energy Phys. 08 (2014) 134.

[31] A. M. Sirunyan et al. (CMS Collaboration), J. High Energy Phys. 09 (2017) 053.

[32] A. M. Iyer and U. Maitra, Phys. Rev. D 95, 035039 (2017).

[33] S. Fichet, G. von Gersdorff, E. Pontn, and R. Rosenfeld, J. High Energy Phys. 01 (2017) 012.

[34] In the following we refer improperly to mesons.

[35] R. Barcelo, A. Carmona, M. Chala, M. Masip, and J. Santiago, Nucl. Phys. B857, 172 (2012).

[36] C. Bini, R. Contino, and N. Vignaroli, J. High Energy Phys. 01 (2012) 157.

[37] K. Kong, M. McCaskey, and G. W. Wilson, J. High Energy Phys. 04 (2012) 079.
[38] N. Vignaroli, Phys. Rev. D 91, 115009 (2015).

[39] J. P. Araque, N. F. Castro, and J. Santiago, J. High Energy Phys. 11 (2015) 120.

[40] K. Agashe, R. Contino, and A. Pomarol, Nucl. Phys. B719, 165 (2005).

[41] M. Chala, Phys. Rev. D 96, 015028 (2017).

[42] G. Aad et al. (ATLAS Collaboration), Phys. Lett. B 758, 249 (2016).

[43] A. Chakraborty, A.M. Iyer, and T.S. Roy, arXiv:1707.07084.

[44] J. A. Aguilar-Saavedra, J. H. Collins, and R. K. Mishra, J. High Energy Phys. 11 (2017) 163.

[45] S. D. Ellis, T. S. Roy, and J. Scholtz, Phys. Rev. D 87, 014015 (2013).

[46] S. D. Ellis, T. S. Roy, and J. Scholtz, Phys. Rev. Lett. 110, 122003 (2013).

[47] B. C. Allanach, D. Bhatia, and A. M. Iyer, Eur. Phys. J. C 77, 595 (2017).

[48] P. Speckmayer, A. Hocker, J. Stelzer, and H. Voss, in Proceedings, 17th International Conference on Computing in High Energy and Nuclear Physics (CHEP 2009), Prague, Czech Republic [J. Phys. Conf. Ser. 219, 032057 (2010)].

[49] J. Alwall, R. Frederix, S. Frixione, V. Hirschi, F. Maltoni, O. Mattelaer, H. S. Shao, T. Stelzer, P. Torrielli, and M. Zaro, J. High Energy Phys. 07 (2014) 079.

[50] N. D. Christensen and C. Duhr, Comput. Phys. Commun. 180, 1614 (2009).

[51] T. Sjostrand, S. Mrenna, and P. Z. Skands, Comput. Phys. Commun. 178, 852 (2008).

[52] J. de Favereau, C. Delaere, P. Demin, A. Giammanco, V. Lematre, A. Mertens, and M. Selvaggi (DELPHES 3 Collaboration), J. High Energy Phys. 02 (2014) 057.

[53] M. Cacciari, G. P. Salam, and G. Soyez, Eur. Phys. J. C 72, 1896 (2012).

[54] M. Cacciari, G. P. Salam, and G. Soyez, J. High Energy Phys. 04 (2008) 063. 